\title{
Videothoracoscopy in the treatment of benign neurogenic tumours of the posterior mediastinum
}

\author{
Mariusz P. Łochowski, Daniel Brzeziński, Józef Kozak \\ Department of Thoracic Surgery, Medical University of Lodz, Lodz, Poland
}

Videosurgery Miniinv 2014; 9 (3): 315-318

DOI: $10.5114 /$ wiitm.2014.44255

\begin{abstract}
Introduction: The indications for videothoracoscopy are very broad and include the treatment of mediastinal tumours.

Aim: To present our experience of using the minimally invasive technique in treating benign neurogenic tumours.

Material and methods: Twenty-two patients were treated due to tumours of the posterior mediastinum from 2003 to 2012. The size of the tumours ranged from $2 \mathrm{~cm}$ to $25 \mathrm{~cm}$. Tumours up to the size of $6 \mathrm{~cm}$ were treated using videothoracoscopy (VT), bigger ones through thoracotomy.

Results: The videothoracoscopy technique was used in 17 patients, thoracotomy in 5. In 2 cases conversion was required due to adhesions in the pleural cavity preventing VT treatment. Complications related to the procedure were not observed. The average time of hospital stay after VT treatment was 4 days, while after thoracotomy it was 6 days. Histologically, tumours of benign nature were found in all cases. Schwannoma was diagnosed in 15 patients, ganglioneuroma in 3 patients, neurofibroma in 3 patients, and chemodectoma in 1 patient. None of the 3 cases of neurofibroma was associated with Recklinghausen's disease. At a mean follow-up of 60 months no recurrence of the tumour was found.

Conclusions: In the case of tumours up to $6 \mathrm{~cm}$ the best surgical technique is videothoracoscopy. In the case of large tumours the best access is the open technique. The minimally invasive technique allows one to shorten the patient's treatment time, reduce postoperative pain and obtain a good cosmetic effect of the treatment.
\end{abstract}

Key words: neurogenic tumours, mediastinum, videothoracoscopy.

\section{Introduction}

Neurogenic tumours occur most often in the posterior mediastinum. Their incidence is estimated at $12-24 \%$ of all primary mediastinal tumours. Malignant forms rarely occur in adults (6\% of cases), while they constitute a significant percentage in children up to 5 years of age ( $40 \%$ of cases). Benign tumours grow slowly and can achieve large sizes, being asymptomatic at the same time [1, 2]. The rapid development of technology in medicine at the end of the $20^{\text {th }}$ century allowed the introduction of new surgical techniques.
Minimally invasive access became possible when formerly only open surgery had been performed [3].

\section{Aim}

In this paper we want to present our own experience of the use of videothoracoscopy in treating benign neurogenic tumours of the mediastinum.

\section{Material and methods}

Twenty-two patients were treated due to tumours of the posterior mediastinum from 2003 to 
2012. There were 15 women and 7 men; the age of the patients ranged from 35 to 68 (mean of 48) years. In $50 \%$ of cases the patients did not report any symptoms and the lesion was detected by accident. The other patients complained of pain in the chest on the side of the tumour. In 1 case (tumour of $25 \mathrm{~cm}$ in diameter occupying the left half of the chest) the patient additionally complained of weakness and shortness of breath. Patients were qualified for the open procedure when the size of the tumour prevented the use of the minimally invasive technique. The sizes of the tumours ranged from $2 \mathrm{~cm}$ to $25 \mathrm{~cm}$. In 17 patients they were oval lesions up to $6 \mathrm{~cm}$ in diameter located in the posterior mediastinum (Photo 1). In the other cases there were large tumours occupying a large part of the pleural cavity (Photo 2).

\section{Results}

The videothoracoscopy technique (VT) was used in 17 patients, while thoracotomy was used in 5 . In 2 patients conversion due to technical difficulties during the VT procedure was necessary (adhesions). All patients were intubated with a double-lumen tube and laid down in the posterolateral position. The first port (video camera) was positioned at the midaxillary line in the fifth or sixth intercostal spaces. The other two ports were most often inserted at the anterior and posterior midaxillary lines in intercostal spaces depending on tumour location. In cases of tumours longer than $6 \mathrm{~cm}$ (giant tumours), anterolateral or posterolateral thoracotomy was performed. All tu-

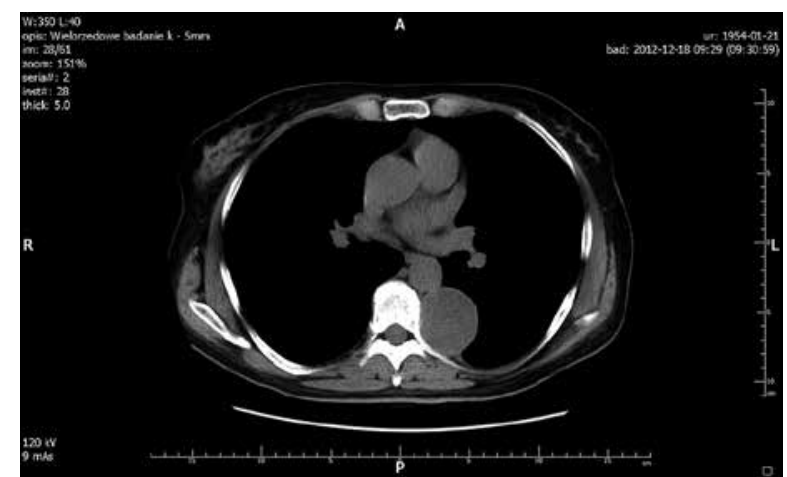

Photo 1. Posterior mediastinal neurofibroma on the left side. The patient was treated with the minimally invasive technique mours were covered by the parietal pleura and connected with the nerve structure. During the VT procedure the pleura was cut with a hook knife, while nerves and vessels were tied up with endo-clips. Special attention was necessary when the tumour was located near the aorta. After the open and VT procedure, one drain was put into the pleural cavity. There were no complications connected with the procedure. In all cases the tumour was located far from the stellate ganglion so pre- and post-operative Horner's syndrome was not observed. Patients after thoracotomy required a longer time of maintaining drainage (4 days) than patients after VT surgery (2 days) and manifested greater pain. The mean length of hospital stay was 4 days after VT treatment and 6 days after thoracotomy. Histologically, benign tumours were confirmed in all cases. The predominant tumour was schwannoma - 15 patients (68\%); ganglioneuroma was diagnosed in 3 patients (14\%), neurofibroma in 3 patients (14\%), and chemodectoma in 1 patient (5\%). Recklinghausen's disease was observed in none of the 3 cases of neurofibroma. At a mean follow-up of 60 months no recurrence of the tumour was found.

\section{Discussion}

Neurogenic tumours are located in the posterior mediastinum in 90-95\% of cases [4, 5]. These are most often benign, slow-growing tumours located along the course of the intercostal or sympathetic nerve $[1,6]$. Other locations of neurogenic tumours, not involving the posterior mediastinum, are also described [7].

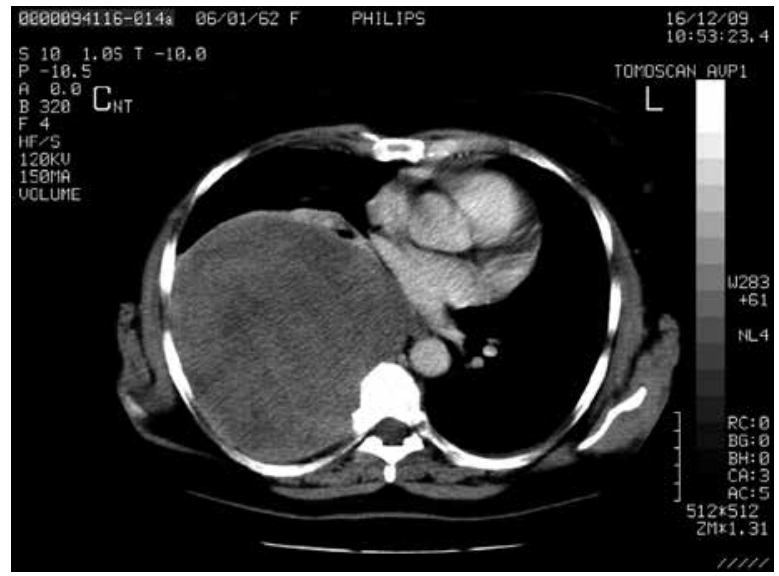

Photo 2. Schwannoma of the posterior mediastinum on the right side. The patient was treated with the open technique 
Benign neurogenic tumours are detected incidentally during routine chest X-rays in 40-60\% of cases $[2,4,8]$. Clinical symptoms occur when the tumour reaches a large size, or when it begins to press against surrounding structures. The most often reported symptoms are cough, shortness of breath, pain in the chest and neurological anomalies [4, 9-11]. In our material $50 \%$ of patients did not present any clinical symptoms. The other patients complained of pain in the chest on the side of the tumour. In 1 case, the patient with a tumour of $25 \mathrm{~cm}$ in diameter additionally complained of shortness of breath and weakness.

Computed tomography (CT) is a common imaging test allowing one to determine the location of the tumour relative to the surrounding structures and plan the surgical access. Performing magnetic resonance imaging $(M R I)$ is recommended in cases when the penetration of the tumour to the spinal canal is suspected $[4,5]$. All our patients had CT of the chest before the surgery. No invasions of the spinal canal were observed.

In order to obtain a good clinical result, the tumour within borders of healthy tissues has to be removed. A commonly preferred surgical access is videothoracoscopy (VT). Shortened hospital stay, lower demand for painkillers and decreased risk of pulmonary complications are the most often mentioned advantages of the minimally invasive technique $[1,2,5,12]$. In our material we used VT in 17 out of 22 patients. Conversions are performed in cases of bleeding or technical difficulties (e.g. pleural adhesions). The frequency of the change of the surgical technique is estimated at $6 \%$ to $22 \%$ in the literature [13]. Two of our patients $(11 \%)$ required the change of the surgical technique due to total obstruction of the pleural cavity. Other complications were not observed. It is not recommended to use minimally invasive techniques in cases when the tumour penetrates into the spinal canal, has a dimension larger than $6 \mathrm{~cm}$ and is located in the top part of the chest. In those particular cases the access of choice is thoracotomy, and, if expansion of the tumour to the spinal canal is found, the thoracic neurosurgery procedure $[1,5$, 13]. In our department patients with tumours over $6 \mathrm{~cm}$ were qualified for the open procedure. The preferred access was mini-thoracotomy. Patients with a tumour of $20 \mathrm{~cm}$ or more in diameter were operated on from the back and side access. After the open procedure the pain persisted approximately a month longer. The average length of hospital stay after minimally invasive surgery is 4 days, while after the open technique it is 6 days. [1] Identical time was noted in our patients. Neurogenic tumours are divided into three important categories: derived from peripheral nerves (nerve sheath tumours), derived from autonomic ganglia (tumours from nerve cells) and derived from paraganglionic cells (paragangliomas). The incidence of those tumours is determined by the patient's age. In adults the most numerous group (83\%) is the group of nerve sheath tumours, among which schwannoma predominates. The tumour is observed in $45-75 \%$ of adult patients [5, 9].

In our material nerve sheath tumours were observed in $82 \%$ of patients, while schwannoma accounted for $68 \%$ of cases. In children the predominant group is the group of tumours derived from nerve cells (69\%), while their malignant forms are often observed in the first 5 years of life $[5,6]$.

The second most common type of nerve sheath tumours is neurofibromas (18\%). In the case of neurofibromatosis (Recklinghausen's disease) the tumour occurs at a young age, may be manifold and more often (30\%) undergoes malignant transformation. In our material no case was associated with neurofibromatosis $[5,9]$.

Benign tumours derived from autonomic ganglion cells (ganglioneuromas) are encapsulated and characterised by high diversity and discreet increase, which often allows the tumour to achieve a large size $[2,9,14]$. In 3 patients treated due to ganglioneuroma in our department, the lesion was detected by accident without clinical symptoms, although the largest dimension of one of the tumours was $20 \mathrm{~cm}$.

The rarest group included paraganglion tumours belonging to the same group as the pheochromocytoma tumour, but not showing endocrine activity. The characteristic features of the tumour are the location in the groove of the thoracic cord and rich vascularity [14]. In our material we encountered only 1 case of such a tumour (chemodectoma).

\section{Conclusions}

In the case of tumours up to $6 \mathrm{~cm}$ the best surgical technique is videothoracoscopy. In the case of large size tumours the best access is the open technique. The minimally invasive technique allows one to shorten the patient's treatment time, reduce pain and obtain a good cosmetic effect. 


\section{References}

1. Cardillo G, Carleo F, Kahali MW, et al. Surgical treatment of benign neurogenic tumours of the mediastinum: single institution report. Eur J Cardiothorac Surg 2008; 34: 1210-4.

2. Yamaguchi M, Yashino I, Fukuyama S, et al. Surgical treatment of neurogenic tumors of the chest. Ann Thorac Cardiovasc Surg 2004; 10: 148-51.

3. Bohanes T, Szkorupa M, Klein J, et al. Videothoracoscopic identification of chondromatous hamartoma of the lung. Videosurgery Miniinv 2013; 8: 152-7.

4. Rahman A, Sedera MA, Mourad IA, et al. Posterior mediastinal tumors: outcome of surgery. J Egypt Natl Canc Inst 2005; 17: 1-8.

5. Topcu S, Alper A, Gülhan E, et al. Neurogenic tumors of the mediastinum: a report of 60 cases. Can Respir J 2000; 7: 261-5.

6. Kang CH, Kim YT, Jean SH, et al. Surgical treatment of malignant mediastinal neurogenic tumors in children. Eur J Cardiothorac Surg 2007; 31: 725-30.

7. Smahi M, Lakranbi M, Ouadnouni Y, et al. Intrathoracic phrenic nerve neurofibroma. Ann Thorac Surg 2011; 91: 57-8.

8. Fierro N, D'ermo G, Di Cola G, et al. Posterior mediastinal schwannoma. Asian Cardiovasc Thorac Ann 2003; 11: 72-3.

9. Duwe VB, Sterman DH, Musami Al. Tumors of the mediastinum. Chest 2005; 128: 2893-9.

10. Georghiou GP, Vinde BA, Boikov O, et al. Giant schwannoma of the posterior mediastinum. Eur I Cardiothorac Surg 2003; 24: 1030.

11. Ondo K, Sugio K, Yano T, et al. Cystic schwannoma presenting as massive hemoptysis in an adult. Respiration 2000; 67: 327-9.

12. Ortigara L, Rosemberg N, Siqueria R, et al. Resection of a mediastinal schwannoma using videoassisted thoracoscopy. J Bras Pneumol 2006; 32: 172-5.

13. Kitami A, Suzuki T, Usuda R, et al. Diagnostic and therapeutic thoracoscopy for mediastinal disease. Ann Thorac Cardiovasc Surg 2004; 10: 14-8.

14. Bousamra M, Wrightson W. Neurogenic mediastinal tumors. Pearson's Thoracic \& Esophageal Surgery. Churchill Livingstone 2008; 1634-40.

Received: 1.10.2013, accepted: 20.04.2014. 